\title{
Update on $\varepsilon_{K}$ with lattice QCD inputs
}

\author{
Yong-Chull Jang ${ }^{23}$, Weonjong Lee ${ }^{1, \star}$, Sunkyu Lee ${ }^{1}$, and Jaehoon Leem ${ }^{1}$ \\ ${ }^{1}$ Lattice Gauge Theory Research Center, CTP, Department of Physics and Astronomy, \\ Seoul National University, Seoul 08826, South Korea \\ ${ }^{2}$ Los Alamos National Laboratory, Theoretical Division T-2, Los Alamos, New Mexico 87545, USA \\ ${ }^{3}$ Brookhaven National Laboratory, Department of Physics, Upton, New York 11973, USA
}

\begin{abstract}
We report updated results for $\varepsilon_{K}$, the indirect $\mathrm{CP}$ violation parameter in neutral kaons, which is evaluated directly from the standard model with lattice QCD inputs. We use lattice QCD inputs to fix $\hat{B}_{K},\left|V_{c b}\right|, \xi_{0}, \xi_{2},\left|V_{u s}\right|$, and $m_{c}\left(m_{c}\right)$. Since Lattice 2016, the UTfit group has updated the Wolfenstein parameters in the angle-only-fit method, and the HFLAV group has also updated $\left|V_{c b}\right|$. Our results show that the evaluation of $\varepsilon_{K}$ with exclusive $\left|V_{c b}\right|$ (lattice QCD inputs) has $4.0 \sigma$ tension with the experimental value, while that with inclusive $\left|V_{c b}\right|$ (heavy quark expansion based on OPE and QCD sum rules) shows no tension.
\end{abstract}

\section{Introduction}

This paper is an update of our previous papers [1-4]. In the standard model, the indirect CP violation parameter $\varepsilon_{K}$ of neutral kaons can be expressed as follows,

$$
\begin{aligned}
\varepsilon_{K} & \equiv \frac{\mathcal{A}\left(K_{L} \rightarrow \pi \pi(I=0)\right)}{\mathcal{A}\left(K_{S} \rightarrow \pi \pi(I=0)\right)} \\
& =e^{i \theta} \sqrt{2} \sin \theta\left(C_{\varepsilon} \hat{B}_{K} X_{\mathrm{SD}}+\frac{\xi_{0}}{\sqrt{2}}+\xi_{\mathrm{LD}}\right)+O\left(\omega \varepsilon^{\prime}\right)+O\left(\xi_{0} \Gamma_{2} / \Gamma_{1}\right),
\end{aligned}
$$

where

$$
\begin{aligned}
C_{\varepsilon} & =\frac{G_{F}^{2} F_{K}^{2} m_{K^{0}} M_{W}^{2}}{6 \sqrt{2} \pi^{2} \Delta M_{K}}, \quad \xi_{0}=\frac{\operatorname{Im} A_{0}}{\operatorname{Re} A_{0}}, \quad \xi_{\mathrm{LD}}=\frac{m_{\mathrm{LD}}^{\prime}}{\sqrt{2} \Delta M_{K}}, \\
m_{\mathrm{LD}}^{\prime} & =-\operatorname{Im}\left[\mathcal{P} \sum_{C} \frac{\left\langle\bar{K}^{0}\left|H_{\mathrm{w}}\right| C\right\rangle\left\langle C\left|H_{\mathrm{w}}\right| K^{0}\right\rangle}{m_{K^{0}}-E_{C}}\right] \\
X_{\mathrm{SD}} & =\operatorname{Im} \lambda_{t}\left[\operatorname{Re} \lambda_{c} \eta_{c c} S_{0}\left(x_{c}\right)-\operatorname{Re} \lambda_{t} \eta_{t t} S_{0}\left(x_{t}\right)-\left(\operatorname{Re} \lambda_{c}-\operatorname{Re} \lambda_{t}\right) \eta_{c t} S_{0}\left(x_{c}, x_{t}\right)\right] .
\end{aligned}
$$

Here, $X_{\mathrm{SD}}$ represents the short distance contribution from the box diagrams. The formulas for $\lambda_{i}, S_{0}$, $x_{i}$, and $\eta_{i j}$ with $i, j=\{c, t\}$ are given in Ref. [1]. The $\xi_{0}$ and $\xi_{\mathrm{LD}}$ represent the long distance effects from the absorptive part and the dispersive part, respectively.

^ Speaker, e-mail: wlee@snu.ac.kr 
Since Lattice 2016, there has been a major update on the Wolfenstein parameters: $\lambda, \bar{\rho}$, and $\bar{\eta}$ from the angle-only fit (AOF) of the UTfit collaboration as well as those from the global unitarity triangle (UT) fit of the CKMfitter and UTfit collaborations. Since Lattice 2016, the HFLAV group has also updated results of $\left|V_{c b}\right|$ and $\left|V_{u b}\right|$. Hence, it is time to update the current status of $\varepsilon_{K}$ in lattice QCD.

\section{Input parameters}

Wolfenstein parameters, $\hat{B}_{K},\left|V_{c b}\right|, \xi_{0}$, and $\xi_{\mathrm{LD}}$ are separately discussed in the following subsections. Other input parameters in Eq. (1) are the same as in Ref. [1] except for the charm quark mass $m_{c}\left(m_{c}\right)=$ 1.2733(76) $\mathrm{GeV}$, which is taken from the HPQCD collaboraion result [5]. They are given in Tables 1 and 2.

\begin{tabular}{clc}
\hline \hline Input & Value & Ref. \\
\hline$\eta_{c c}$ & $1.72(27)$ & {$[1]$} \\
\hline$\eta_{t t}$ & $0.5765(65)$ & {$[6]$} \\
\hline$\eta_{c t}$ & $0.496(47)$ & {$[7]$} \\
\hline \hline
\end{tabular}

Table 1. QCD corrections: $\eta_{c c}, \eta_{t t}$, and $\eta_{c t}$.

Table 2. Other input parameters.

\begin{tabular}{clc}
\hline \hline Input & Value & Ref. \\
\hline$G_{F}$ & $1.1663787(6) \times 10^{-5} \mathrm{GeV}^{-2}$ & {$[8]$} \\
\hline$M_{W}$ & $80.385(15) \mathrm{GeV}$ & {$[8]$} \\
\hline$m_{c}\left(m_{c}\right)$ & $1.2733(76) \mathrm{GeV}$ & {$[5]$} \\
\hline$m_{t}\left(m_{t}\right)$ & $163.3(2.7) \mathrm{GeV}$ & {$[9]$} \\
\hline \hline
\end{tabular}

(a)

\begin{tabular}{clc}
\hline \hline Input & Value & Ref. \\
\hline$\theta$ & $43.52(5)^{\circ}$ & {$[8]$} \\
\hline$m_{K^{0}}$ & $497.614(24) \mathrm{MeV}$ & {$[8]$} \\
\hline$\Delta M_{K}$ & $3.484(6) \times 10^{-12} \mathrm{MeV}$ & {$[8]$} \\
\hline$F_{K}$ & $156.2(7) \mathrm{MeV}$ & {$[8]$} \\
\hline \hline
\end{tabular}

(b)

\subsection{Wolfenstein parameters}

Table 3. Wolfenstein parameters.

\begin{tabular}{c|ccc}
\hline \hline & CKMfitter & UTfit & AOF $[10]$ \\
\hline$\lambda$ & $0.22509(29) /[11]$ & $0.22497(69) /[12]$ & $0.2248(6) /[13]$ \\
\hline $\bar{\rho}$ & $0.1598(76) /[11]$ & $0.153(13) /[12]$ & $0.146(22) /[14]$ \\
\hline $\bar{\eta}$ & $0.3499(63) /[11]$ & $0.343(11) /[12]$ & $0.333(16) /[14]$ \\
\hline \hline
\end{tabular}

The CKMfitter and UTfit collaboration provide the Wolfenstein parameters $(\lambda, \bar{\rho}, \bar{\eta}, A)$ determined by the global unitarity triangle (UT) fit. The 2017 results are summarized in Table 3 . Here, $\varepsilon_{K}, \hat{B}_{K}$, and $\left|V_{c b}\right|$ are used as inputs to the global UT fit. Hence, the Wolfenstein parameters extracted by the global UT fit contain unwanted correlation with $\varepsilon_{K}$. In order to avoid this correlation, we take another input set from the angle-only fit (AOF) suggested in Ref. [10]. The AOF does not use $\varepsilon_{K}, \hat{B}_{K}$, and $\left|V_{c b}\right|$ as input to determine the UT apex $(\bar{\rho}, \bar{\eta})$. We can determine $\lambda$ independently from $\left|V_{u s}\right|$ which comes from the $K_{\ell 3}$ and $K_{\ell 2}$ decays using lattice QCD inputs. The 2017 results of the AOF are summarized in Table 3. 


\subsection{Input parameter $\hat{B}_{K}$}

Recently, FLAG has reported results for $\hat{B}_{K}$ calculated in lattice QCD with $N_{f}=2+1$ flavors [15].

$$
\hat{B}_{K}=0.7625(97) \quad \text { from FLAG-2017. }
$$

This is the global average over the results of BMW-2011 [16], Laiho-2011 [17], RBC-UK-2016 [18], and SWME-2016 [19].

\subsection{Input parameter $\left|V_{c b}\right|$}

Table 4. Results for $\left|V_{c b}\right|$ in units of $1.0 \times 10^{-3}$

\begin{tabular}{l|l|l}
\hline \hline$B \rightarrow D^{*} \ell \bar{v}$ & $39.05(47)(58)$ & [20] HFLAV 2017 \\
\hline$B \rightarrow D \ell \bar{v}$ & $39.18(94)(36)$ & [20] HFLAV 2017 \\
\hline$\left|V_{u b}\right| /\left|V_{c b}\right|$ & $0.080(4)(4)$ & [20] HFLAV 2017 \\
\hline \hline ex-combined & $39.13(59)$ & [20] HFLAV 2017 \\
\hline \hline
\end{tabular}

(a) Exclusive $\left|V_{c b}\right|$

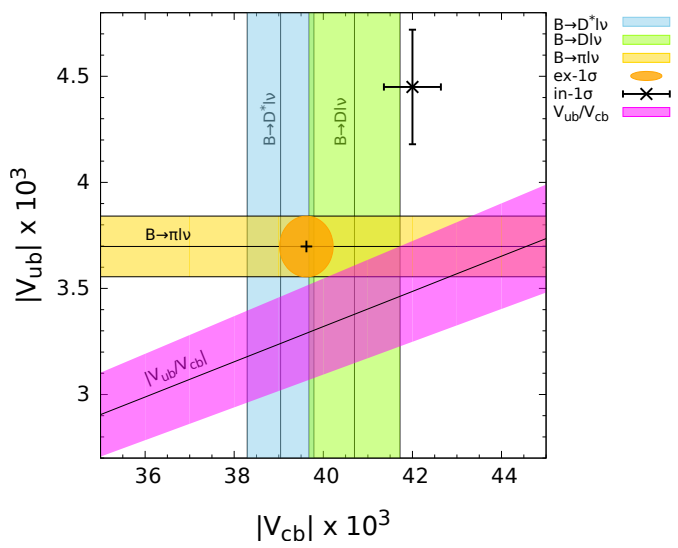

(a) Lattice 2016

\begin{tabular}{l|l|l}
\hline \hline kinetic scheme & $42.19(78)$ & [20] HFLAV 2017 \\
\hline 1S scheme & $41.98(45)$ & [20] HFLAV 2017 \\
\hline \hline in-combined & $42.03(39)$ & this paper \\
\hline \hline
\end{tabular}

(b) Inclusive $\left|V_{c b}\right|$

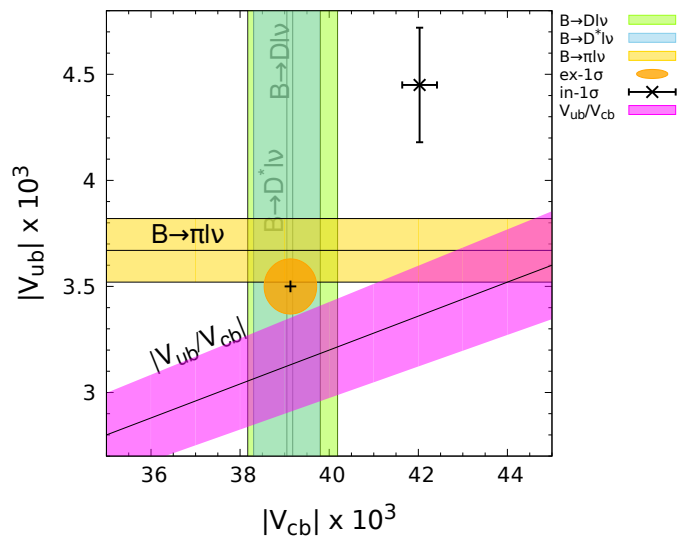

(b) Lattice 2017

Figure 1. $\left|V_{c b}\right|$ versus $\left|V_{u b}\right|$ in units of $1.0 \times 10^{-3}$ as of (a) Lattice $2016[3,4]$ and (b) Lattice 2017 [20]: The light-blue band represents $\left|V_{c b}\right|$ determined from the $\bar{B} \rightarrow D^{*} \ell \bar{v}$ decay mode. The light-green band represents $\left|V_{c b}\right|$ determined from the $\bar{B} \rightarrow D \ell \bar{v}$ decay mode. The yellow band represents $\left|V_{u b}\right|$ determined from the $\bar{B} \rightarrow \pi \ell \bar{v}$ decay mode. The magenta band represents $\left|V_{u b} / V_{c b}\right|$ determined from the LHCb data of the $\Lambda_{b} \rightarrow \Lambda_{c} \ell \bar{v}$ and $\Lambda_{b} \rightarrow p \ell \bar{v}$ decay modes. The orange circle represents the combined results for exclusive $\left|V_{c b}\right|$ and $\left|V_{u b}\right|$ from the $B$ meson and $\Lambda_{b}$ decays within $1.0 \sigma$. The black cross $(\mathbf{x})$ represents the inclusive $\left|V_{c b}\right|$ and $\left|V_{u b}\right|$ from the heavy quark expansion. The details are given in Table 4.

In Table 4, we summarize updated results for both exclusive $\left|V_{c b}\right|$ and inclusive $\left|V_{c b}\right|$. Recently HFLAV reported them [20]. At present, we find that there exists a $4.1 \sigma$ tension between exclusive 
and inclusive $\left|V_{c b}\right|$ when we use the combined averages given in Table 4. We use these combined results when we evaluate $\varepsilon_{K}$.

In Fig. 1, we present results for $\left|V_{c b}\right|$ and $\left|V_{u b}\right|$. The big change is that, as of Lattice 2016, the result for exclusive $\left|V_{c b}\right|$ from $\bar{B} \rightarrow D \ell \bar{v}$ was about one sigma away from that from $\bar{B} \rightarrow D^{*} \ell \bar{v}$ (refer to Ref. [3, 4] for more details), but as of Lattice 2017, they are on top of each other, as shown in Fig. 1 (b). In 2017, due to the addition of more data from ALEPH, CLEO, OPAL, and DELPHI to the HFLAV analysis for exclusive $\left|V_{c b}\right|$, the results from $\bar{B} \rightarrow D^{*} \ell \bar{v}$ do not change visibly, but those from $\bar{B} \rightarrow D \ell \bar{v}$ shift downward by about $1.0 \sigma$. For more details, refer to Ref. [20].

Regarding $\left|V_{u b}\right| /\left|V_{c b}\right|$, in 2016, we used the results of lattice QCD in Ref. [21], but in 2017, we use the results of HFLAV in Ref. [20]. Due to the addition of more data to the HFLAV analysis, the results shift downward by $\frac{3}{4} \sigma$ in 2017 .

Regarding $\left|V_{u b}\right|$, in 2016, we used the results of Ref. [22] obtained using a combined fit of the results in Refs. [23, 24] for $B \rightarrow \pi \ell v$ decay over the data subset from BABAR and BELLE, but in 2017, we use the results of HFLAV in Ref. [20] obtained using the exclusive decay results of $B \rightarrow \pi \ell v$ from BABAR and BELLE. Both results are used as input to determine $\left|V_{c b}\right|$ and $\left|V_{u b}\right|$ from the combined fit with all the exclusive decay results for $B$ mesons and $\Lambda_{b}$ baryons. The shift in $\left|V_{u b}\right|$ is downward by about $0.1 \sigma$ in 2017 .

\subsection{Input parameters $\xi_{0}$ and $\xi_{\mathrm{LD}}$}

The absorptive part of long distance effects in $\varepsilon_{K}$ is parametrized into $\xi_{0}$. It is related to $\varepsilon^{\prime} / \varepsilon$ and $\xi_{2}$ as follows,

$$
\xi_{0}=\frac{\operatorname{Im} A_{0}}{\operatorname{Re} A_{0}}, \quad \xi_{2}=\frac{\operatorname{Im} A_{2}}{\operatorname{Re} A_{2}}, \quad \operatorname{Re}\left(\frac{\varepsilon^{\prime}}{\varepsilon}\right)=\frac{\omega}{\sqrt{2}\left|\varepsilon_{K}\right|}\left(\xi_{2}-\xi_{0}\right) .
$$

There are two independent methods to determine $\xi_{0}$ in lattice QCD: one is the indirect method and the other is the direct method. In the indirect method, we determine $\xi_{0}$ using Eq. (6) with lattice QCD input $\xi_{2}$ and with experimental results for $\varepsilon^{\prime} / \varepsilon, \varepsilon_{K}$, and $\omega$. In the direct method, we can determine $\xi_{0}$ directly using lattice QCD results for $\operatorname{Im} A_{0}$ combined with experimental results for $\operatorname{Re} A_{0}$. Here, we prefer the indirect method to the direct method thanks to two reasons. The first reason is that the lattice QCD calculation of $\operatorname{Im} A_{0}$ is much noisier than that of $\operatorname{Im} A_{2}$ due to many disconnected diagrams. The second reason is that the S-wave phase shift $\delta_{0}$ of the $\pi-\pi$ scattering in Ref. [25] is lower by $3.0 \sigma$ than the conventional determination of $\delta_{0}$ in Ref. [26-28], which indicates that there might be some issues unresolved at present. For more details on the second reason, refer to Ref. [3, 4]. In Table 5, we present results of $\xi_{0}$ determined using both indirect and direct methods. Here, we use the value of $\xi_{0}$ with the indirect method.

\begin{tabular}{llll}
\hline \hline Input & Method & Value & Ref. \\
\hline$\xi_{0}$ & indirect & $-1.63(19) \times 10^{-4}$ & {$[29]$} \\
\hline$\xi_{0}$ & direct & $-0.57(49) \times 10^{-4}$ & {$[25]$} \\
\hline$\xi_{\mathrm{LD}}$ & - & $(0 \pm 1.6) \%$ & {$[30]$} \\
\hline \hline
\end{tabular}

Table 5. Long distance effects: $\xi_{0}$ and $\xi_{\text {LD }}$. In the case of $\xi_{\mathrm{LD}}$, we do not know the precise value at present, and so we quote a rough estimate [31] incorporated as a systematic error.

The $\xi_{\mathrm{LD}}$ parameter represents the long distance effect from the dispersive part. There has been an on-going attempt to calculate it in lattice QCD [31]. However, this attempt [32] is an exploratory study rather than a high precision measurement at present. Hence, in this paper we use the rough estimate of $\xi_{\mathrm{LD}}$ in Ref. [31]. It is given in Table 5. 


\section{Results for $\varepsilon_{K}$ with lattice QCD inputs}

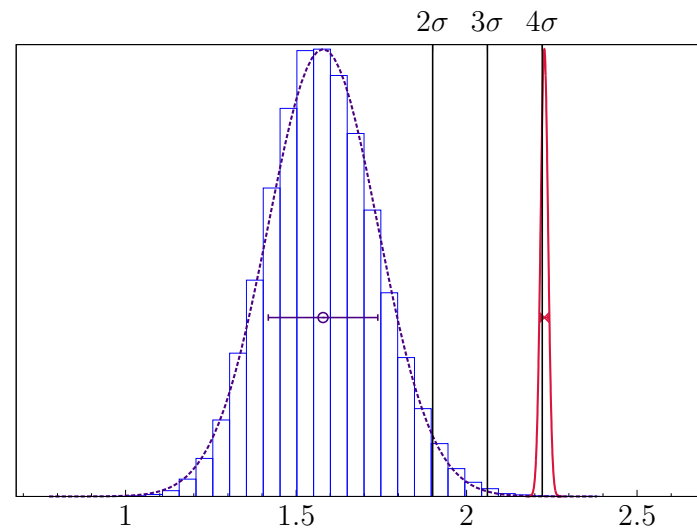

(a) $\left|\varepsilon_{K}\right|$ with exclusive $\left|V_{c b}\right|$

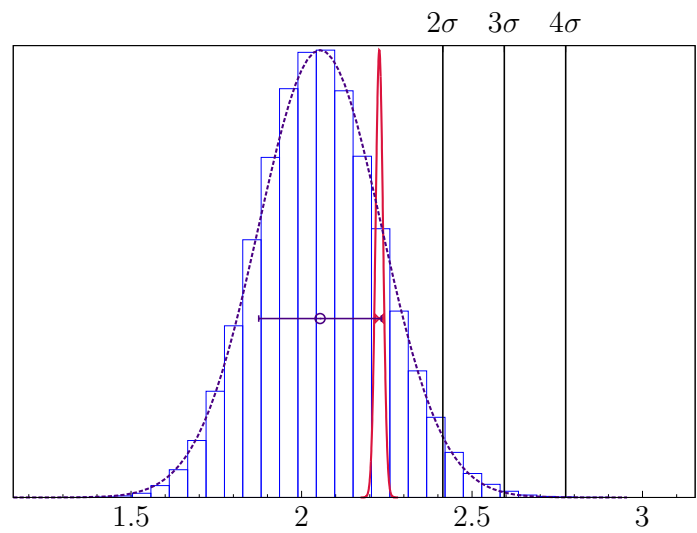

(b) $\left|\varepsilon_{K}\right|$ with inclusive $\left|V_{c b}\right|$

Figure 2. $\left|\varepsilon_{K}\right|$ with (a) exclusive $\left|V_{c b}\right|$ (left) and (b) inclusive $\left|V_{c b}\right|$ (right) in units of $1.0 \times 10^{-3}$. Here, we use the FLAG-2017 $\hat{B}_{K}$ and AOF for the Wolfenstein parameters. The red curve represents the experimental value of $\varepsilon_{K}$, and the blue curve represents the theoretical value calculated directly from the standard model.

In Fig. 2, we present results for $\varepsilon_{K}$ calculated directly from the standard model with the lattice QCD inputs described in the previous section. In Fig. 2(a), the blue curve represents the theoretical evaluation of $\varepsilon_{K}$ with FLAG $\hat{B}_{K}$, AOF Wolfenstein parameters, and exclusive $\left|V_{c b}\right|$ which corresponds to ex-combined in Table 4 (a). The red curve in Fig. 2 represents the experimental value of $\varepsilon_{K}$. In Fig. 2 (b), the blue curve represents the same as in Fig. 2 (a) except for using the inclusive $\left|V_{c b}\right|$ which corresponds to in-combined in Table 4 (b).

The updated results for $\varepsilon_{K}$ are, in units of $1.0 \times 10^{-3}$,

$$
\begin{aligned}
& \left|\varepsilon_{K}\right|=1.58 \pm 0.16 \quad \text { for exclusive }\left|V_{c b}\right| \text { (lattice QCD) } \\
& \left|\varepsilon_{K}\right|=2.05 \pm 0.18 \quad \text { for inclusive }\left|V_{c b}\right| \text { (QCD sum rule) } \\
& \left|\varepsilon_{K}\right|=2.228 \pm 0.011 \quad \text { (experimental value) }
\end{aligned}
$$

This indicates that the theoretical evaluation of $\varepsilon_{K}$ with lattice QCD inputs (with exclusive $\left|V_{c b}\right|$ ) has $4.0 \sigma$ tension with the experimental result, while there is no tension in the inclusive $\left|V_{c b}\right|$ channel (heavy quark expansion based on the OPE and QCD sum rules).

In Table 6 , we present the error budget for $\varepsilon_{K}^{\mathrm{SM}}$. Here, we find that the uncertainty from $\left|V_{c b}\right|$ is dominant in the error budget, while the errors from $\bar{\eta}$ and $\eta_{c t}$ are sub-dominant. Hence, if we are to see a gap $\Delta \varepsilon_{K}$ greater than $5.0 \sigma$, it is essential to reduce the error in $\left|V_{c b}\right|$ significantly. To reduce this error as much as possible, a project to calculate $\bar{B} \rightarrow D^{(*)} \ell \bar{v}$ form factors using the OK action is underway. Note that the OK action is improved up to the $\lambda^{3} \operatorname{order}^{1}$ in the HQET power counting, while the original Fermilab action is improved up to the $\lambda^{1}$ order. For more details on this issue, refer to Refs. [33-36].

In Fig. 3, we plot the $\Delta \varepsilon_{K}=\varepsilon_{K}^{\text {Exp }}-\varepsilon_{K}^{\mathrm{SM}}$ in units of $\sigma$ (= the total error in the estimate of $\Delta \varepsilon_{K}$ ) as the time evolves starting from 2012. We started to monitor $\Delta \varepsilon_{K}$ in 2012 when several lattice QCD

$$
{ }^{1} \lambda \approx \frac{\Lambda}{2 m_{Q}} \approx \frac{1}{8} \text { for the charm quark. }
$$




\begin{tabular}{ccc}
\hline \hline source & error $(\%)$ & memo \\
\hline$V_{c b}$ & 30.1 & ex-combined \\
$\bar{\eta}$ & 25.8 & AOF \\
$\eta_{c t}$ & 20.8 & $c-t$ Box \\
$\eta_{c c}$ & 8.8 & $c-c$ Box \\
$\bar{\rho}$ & 3.9 & AOF \\
$m_{t}$ & 3.0 & \\
$\xi_{\mathrm{LD}}$ & 2.5 & RBC/UKQCD \\
$\hat{B}_{K}$ & 1.8 & FLAG-2017 \\
$\xi_{0}$ & 1.3 & RBC/UKQCD \\
$\vdots$ & $\vdots$ & \\
\hline \hline
\end{tabular}

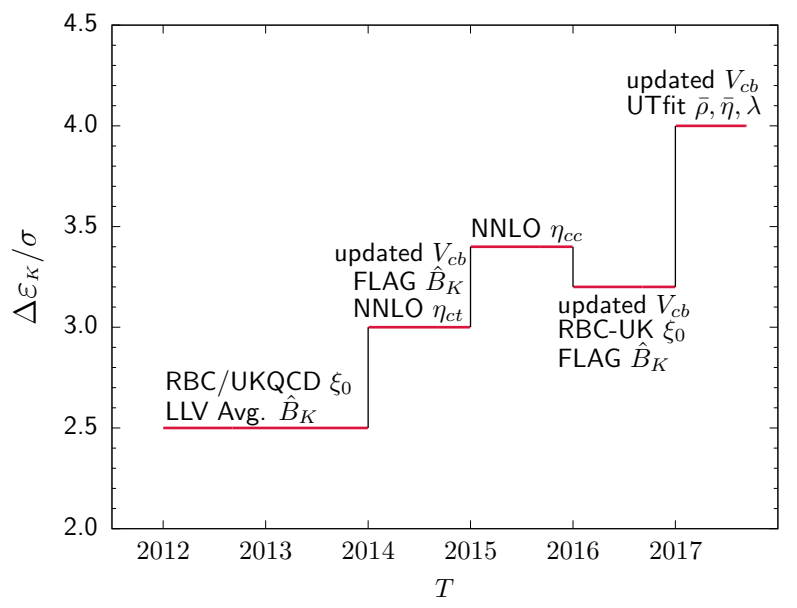

Table 6. Error budget for $\varepsilon_{K}^{\mathrm{SM}}$ obtained using the AOF method, the exclusive $\left|V_{c b}\right|$, and the FLAG $\hat{B}_{K}$. Here, the values in the error column are fractional contributions in units of percentage $(\%)$ to the total error obtained using the formula given in Ref. [1]. Hence, the total sum of the errors( $\%$ ) will be $100 \%$.

Figure 3. Chronological evolution of $\Delta \varepsilon_{K}$ in units of $\sigma$. Here, $\Delta \varepsilon_{K} \equiv \varepsilon_{K}^{\text {Exp }}-\varepsilon_{K}^{\text {SM }}$, where $\varepsilon_{K}^{\text {Exp }}$ represents the experimental value of $\varepsilon_{K}$, and $\varepsilon_{K}^{\mathrm{SM}}$ represents the theoretical evaluation of $\varepsilon_{K}$ calculated directly from the standard model with lattice QCD inputs. Here, $1.0 \sigma$ represents the statistical and systematic uncertainty in the estimation of $\Delta \varepsilon_{K}$.

results for $\hat{B}_{K}$ obtained using different methods became consistent with each other within one sigma. In $2012, \Delta \varepsilon_{K}$ was $2.5 \sigma$ but now it is $4.0 \sigma$. To understand the time evolution over the past 5 years, we have performed an additional analysis on the error and average.

In Fig. 4 (a), we plot the chronological evolution of $\Delta \varepsilon_{K}$ and its error $\sigma_{\Delta \varepsilon_{K}}$. Here, we find that the value for $\Delta \varepsilon_{K}$ has increased with some fluctuations by $25 \%$ during the period of 2012-2017, and its error $\sigma_{\Delta \varepsilon_{K}}$ has decreased monotonically by $24 \%$ in the same period. These two effects interfere constructively so as to produce the $4.0 \sigma$ tension in $\Delta \varepsilon_{K}$ in 2017. The monotonic decrease in the error $\sigma_{\Delta \varepsilon_{K}}$ reflects the fact that lattice QCD calculations are becoming more precise, and the experimental results also are becoming more accurate. In Fig. 4 (b), we show the time evolution of $\varepsilon_{K}^{\mathrm{SM}}$ and $\Delta \varepsilon_{K}$ in units of $\varepsilon_{K}^{\mathrm{Exp}}$. Here, we find that the evaluation of $\varepsilon_{K}^{\mathrm{SM}}$ with lattice QCD inputs explains only $71 \%$ of the experimental value, and the gap of about $29 \%$ in $\Delta \varepsilon_{K}$ cannot be described in the standard model with lattice QCD inputs.

\section{Acknowledgement}

We would like to express our sincere gratitude to Jon Bailey for help with the manuscript. We would like to express sincere gratitude to Carleton DeTar, Aida El-Khadra, and Andreas Kronfeld for helpful discussion. We also would like to express sincere gratitude to Guido Martinelli for providing to us most updated results of 


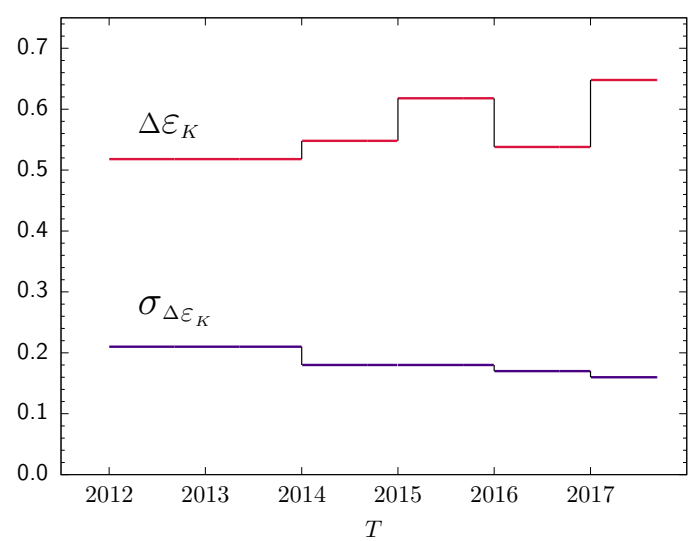

(a) history of $\Delta \varepsilon_{K}$ and $\sigma_{\Delta \varepsilon_{K}}$

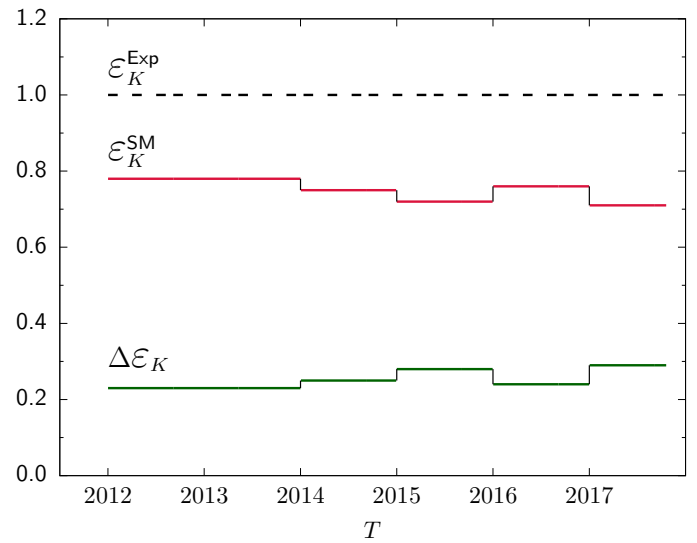

(b) history of $\varepsilon_{K}^{\mathrm{SM}}$, and $\Delta \varepsilon_{K}$

Figure 4. Chronological evolution of (a) $\Delta \varepsilon_{K}$ and $\sigma_{\Delta \varepsilon_{K}}$ in units of $1.0 \times 10^{-3}$, and (b) $\varepsilon_{K}^{\mathrm{SM}}$ and $\Delta \varepsilon_{K}$ in units of $\varepsilon_{K}^{\mathrm{Exp}}$. Here, $\Delta \varepsilon_{K} \equiv \varepsilon_{K}^{\mathrm{Exp}}-\varepsilon_{K}^{\mathrm{SM}}$, where $\varepsilon_{K}^{\mathrm{Exp}}$ represents the experimental value of $\varepsilon_{K}$, and $\varepsilon_{K}^{\mathrm{SM}}$ represents the theoretical evaluation of $\varepsilon_{K}$ calculated directly from the standard model with lattice QCD inputs. Here, $\sigma_{\Delta \varepsilon_{K}}$ represents the statistical and systematic uncertainty in the estimation of $\Delta \varepsilon_{K}$.

UTfit. The research of W. Lee is supported by the Creative Research Initiatives Program (No. 2017013332) of the NRF grant funded by the Korean government (MEST). W. Lee would like to acknowledge the support from the KISTI supercomputing center through the strategic support program for the supercomputing application research [No. KSC-2014-G2-002]. Computations were carried out in part on the DAVID GPU clusters at Seoul National University. J.A.B. is supported by the Basic Science Research Program of the National Research Foundation of Korea (NRF) funded by the Ministry of Education (No. 2014027937).

\section{References}

[1] J.A. Bailey, Y.C. Jang, W. Lee, S. Park (SWME), Phys. Rev. D92, 034510 (2015), 1503.05388

[2] J.A. Bailey, Y.C. Jang, W. Lee, S. Park, PoS LATTICE2015, 348 (2015), 1511.00969

[3] W. Lee, J. Phys. Conf. Ser. 800, 012006 (2017), 1611.04261

[4] J.A. Bailey, W. Lee, J. Leem, S. Park, Y.C. Jang, PoS LATTICE2016, 383 (2016), 1611.00503

[5] B. Chakraborty, C.T.H. Davies, B. Galloway, P. Knecht, J. Koponen, G.C. Donald, R.J. Dowdall, G.P. Lepage, C. McNeile, Phys. Rev. D91, 054508 (2015), 1408. 4169

[6] A.J. Buras, D. Guadagnoli, Phys.Rev. D78, 033005 (2008), 0805. 3887

[7] J. Brod, M. Gorbahn, Phys.Rev. D82, 094026 (2010), 1007.0684

[8] K. Olive et al., Chin.Phys. C38, 090001 (2014)

[9] S. Alekhin, A. Djouadi, S. Moch, Phys.Lett. B716, 214 (2012), 1207.0980

[10] A. Bevan, M. Bona, M. Ciuchini, D. Derkach, E. Franco et al., Nucl.Phys.Proc.Suppl. 241-242, 89 (2013)

[11] J. Charles, A. Hocker, H. Lacker, S. Laplace, F.R. Le Diberder, J. Malcles, J. Ocariz, M. Pivk, L. Roos (CKMfitter Group), Eur. Phys. J. C41, 1 (2005), preliminary results as of ICHEP 2016, http://ckmfitter.in2p3.fr/, hep-ph/0406184 
[12] M. Bona et al. (UTfit), JHEP 10, 081 (2006), Standard Model fit results: Summer 2016 (ICHEP 2016): http://www.utfit.org, hep-ph/0606167

[13] C. Patrignani et al. (Particle Data Group), Chin. Phys. C40, 100001 (2016), https://pdg.lbl.gov/

[14] G. Martinelli et al., Private communication with UTfit, http://www.utfit.org/UTfit/ (2017)

[15] S. Aoki et al., Eur. Phys. J. C77, 112 (2017), 1607.00299

[16] S. Durr, Z. Fodor, C. Hoelbling et al., Phys.Lett. B705, 477 (2011), 1106.3230

[17] J. Laiho, R.S. Van de Water, PoS LATTICE2011, 293 (2011), 1112.4861

[18] T. Blum et al. (RBC, UKQCD), Phys. Rev. D93, 074505 (2016), 1411.7017

[19] B.J. Choi et al. (SWME), Phys. Rev. D93, 014511 (2016), 1509.00592

[20] Y. Amhis et al. (2016), online update at http://www.slac.stanford.edu/xorg/hflav, 1612.07233

[21] W. Detmold, C. Lehner, S. Meinel, Phys. Rev. D92, 034503 (2015), 1503.01421

[22] C. DeTar, in Proceedings of the 27th International Symposium on Lepton Photon Interactions at High Energies (2015), 1511.06884

[23] J.A. Bailey et al. (Fermilab Lattice, MILC), Phys. Rev. D92, 014024 (2015), 1503.07839

[24] J.M. Flynn, T. Izubuchi, T. Kawanai, C. Lehner, A. Soni, R.S. Van de Water, O. Witzel, Phys. Rev. D91, 074510 (2015), 1501.05373

[25] Z. Bai et al. (RBC, UKQCD), Phys. Rev. Lett. 115, 212001 (2015), 1505.07863

[26] G. Colangelo, J. Gasser, H. Leutwyler, Nucl. Phys. B603, 125 (2001), hep-ph/0103088

[27] R. Garcia-Martin, R. Kaminski, J.R. Pelaez, J. Ruiz de Elvira, F.J. Yndurain, Phys. Rev. D83, 074004 (2011), 1102.2183

[28] S. Descotes-Genon, N.H. Fuchs, L. Girlanda, J. Stern, Eur. Phys. J. C24, 469 (2002), hep-ph/0112088

[29] T. Blum et al., Phys. Rev. D91, 074502 (2015), 1502 . 00263

[30] N. Christ, T. Izubuchi, C. Sachrajda, A. Soni, J. Yu, Phys.Rev. D88, 014508 (2013), 1212 . 5931

[31] N. Christ, T. Izubuchi, C.T. Sachrajda, A. Soni, J. Yu, PoS LATTICE2013, 397 (2014), 1402.2577

[32] N.H. Christ, Z. Bai, PoS LATTICE2015, 342 (2016)

[33] J.A. Bailey, T. Bhattacharya, R. Gupta, Y.C. Jang, W. Lee, J. Leem, S. Park, B. Yoon (LANLSWME), Calculation of $\bar{B} \rightarrow D^{*} \ell \bar{v}$ form factor at zero-recoil using the Oktay-Kronfeld action, in Proceedings, 35th International Symposium on Lattice Field Theory (Lattice2017): Granada, Spain, to appear in EPJ Web Conf.

[34] J.A. Bailey, Y.C. Jang, W. Lee, J. Leem (LANL-SWME), Improvement of heavy-heavy current for calculation of $\bar{B} \rightarrow D^{(*)} \ell \bar{v}$ form factors using Oktay-Kronfeld heavy-quark, in Proceedings, 35th International Symposium on Lattice Field Theory (Lattice2017): Granada, Spain, to appear in EPJ Web Conf.

[35] J.A. Bailey, J. Leem, W. Lee, Y.C. Jang, PoS LATTICE2016, 285 (2016), 1612 . 09081

[36] H. Jeong, W. Lee, J. Leem, S. Park, T. Bhattacharya, R. Gupta, Y.C. Jang (LANL-SWME), PoS LATTICE2016, 380 (2016), 1612 . 05707 